\title{
Integrating Gender and Ethnicity in Mobile Courses Ante-Design: a TELearning Instrument
}

\author{
$\underline{\text { doi:10.3991/ijim.v3i1.674 }}$ \\ I. de Waard and M. Zolfo \\ Institute of Tropical Medicine, eLearning, Antwerp, Belgium
}

\begin{abstract}
This paper proposes an instrument to allow instructional designers and subject matter experts (SMEs) to balance gender and ethnicity categories from inception of mobile courses throughout the complete design of the course. This instrument can also be used to analyze the matter of gender and ethnicity in all Technology Enhanced Learning courses.
\end{abstract}

Index Terms - gender, ethnicity, instructional design, mobile learning, quality assurance, technology enhanced learning.

\section{INTRODUCTION}

Although the gender issue is mentioned in many evaluation papers focusing on the design of mobile courses, it is usually mentioned in the post-development stage. This omits the possibility to adjust the depiction or presence of gender matter during the design process of a course. The attention that is given to depiction of both gender and ethnicity will increase as the mobile learning market is expanding to low resource or emerging countries now. This focus is also due to the fact that gender is one of the United Nations Millennium Goals for 2015.

Apart from gender, ethnicity is even less mentioned in reference to the instructional design of mobile courses or eLearning courses in general.

The authors of the paper underline the importance of addressing both gender and ethnicity as a part of the instructional design process of mobile courses, because it is directly linked to the context and identity of the learner.

\section{PROBLEM}

Mobile courses in particular and Technology Enhanced Learning in general have grown in the past few years. Research has pointed out that context and identity are important motivational factors in mobile learning [1]. Gender and ethnicity are part of both context and identity of the learner.

Although gender and ethnicity are looked at in a lot of evaluations, these evaluations are mainly post-design or post-launch of the mobile courses. Little has been done to build an instrument that would allow ante-design or antedevelopment evaluation of both gender and ethnicity.

Ethnicity is getting increasingly important as TELearning goes global. A lot of the mobile courses that are currently available have been designed in developed countries, which reflects in the visuals and the actors' choice that are used in the courses.

With this instrument we hope to deliver a tool allowing researcher to balance and anticipate gender and cultural issues ante- and during development of mobile courses. In doing so it hopes to bring contextual bridges towards mobile learners worldwide.

\section{BACKGROUND}

The social cognitive perspective in teaching and learning emphasizes the importance that social interaction plays in contributing to motivational outcomes such as learner self-efficacy and self-regulation. According to Bandura [2], attribute similarities between a social model and a learner, such as gender, ethnicity, and competency, often have predictive significance for the learner's efficacy beliefs and achievements [3].

Interactive multimedia instructional design is anchored in culture through various world views, selective instructional design paradigms, and learning theories. As such, it is culturally contextualized [4]. Having proactive instructional design is particularly significant for learners who belong to cultures that are situated in an unequal relationship with the dominant groups and consequently have a history of educational failure [4].

In order to be inclusive, a balanced presentation of diverse human groups is required, for example men and women, people with different ethnic and cultural backgrounds or religions, etc. The same argument is made with respect to digital educational materials. Analyses, however, show that such a balanced representation is not always provided [5]. Many authors also point out that it is important to look beyond the mere presence of different groups. Women and blacks are often presented in stereotypical roles and exhibit stereotypical behaviors, and social groups are not always presented in a way that accurately reflects their contributions to a certain area [5].

\section{DESIGN OF THE INSTRUMENT}

The instrument is designed as an easy to use checklist that enables both instructional designers and subject matter experts (SMEs) to quickly evaluate the gender and ethnicity representation build in mobile courses.

The checklist has two big categories (gender ethnicity) and zooms in on relevant actions of the represented personae (e.g., does the person have an active role? Is the person depicted in a stereotypical gender situation?). Each checklist option has additional information to clarify the meaning of the box, if needed.

In addition to the visual representation, we also included the narrator's voice as an option. The option of the narrator's voice is limited to 'female voice', 'male voice' or 'other' (e.g.: if the voice is an impression of an animal that narrates the course and the gender of that voice is not clear). 
All options have either an additional 'other' option or a 'not applicable' option to allow correct input.

The checklist is immediately linked to a database. The results of that database can be revised according to the preferences and the chosen relevance of the person using this checklist to analyze specific courses, per example.

By checking the relevant box(es) while building a course, the instructional designer or SME gets a clear idea of the main focus of the used imagery and/or voices and the roles those images depict. The results of the checklist are easily extrapolated into meaningful statistics that can be used to further develop the courses and ensure a gender and cultural balance throughout the visuals. In case balance is not the goal of the course, than a specific goal for both pictures and/or audio can be taken into account and checked (e.g., if a course were to address people in one specific minority group, the developers could decide that everyone in the visuals should be part of that specific group to ensure empowerement of that specific minority.

With this instrument the Institute of Tropical Medicine (ITM) in Antwerp hopes to deliver a tool allowing developers to anticipate diverse gender and cultural representation ante- and during development of mobile courses

\section{Evaluations}

\section{A. Ethnicity is a sensitive topic}

Although the instrument is still being adapted to better fit the goal, it is clear that making a list of ethnicities is a very difficult process.

During the first tests of this instrument, we choose to limit ethnicity to 'color of skin' as it was a visual criterium and it is frequently visible on many of ITM's images because of our international physicians and due to the medical diagnosis which features skin a lot as well. The categories in the 'color of skin'-option were limited as well, but with an option to add colors. We placed the colors in alphabetical order i.e.: black, brown...

Although ITM is very sensitive towards the term ethnicity, we could not come to an agreement that satisfied all stakeholders involved in developing the courses. Ethnicity needs to be researched to be able to make an objective argument on why certain ethnicities would be taken up or be left out, or certain criteria would be mentioned or not.

\section{B. Enhanced awareness}

The simple fact of having to think about gender and ethnic representation in the courses gave an extra reflective dimension to both the SMEs and the instructional designers. During the testing phase of the checklist users became more aware of their assumptions on balanced representations. Being able to put numbers on the representation, gave the course developers a deeper understanding of both their hidden assumptions and the actual representations of both gender and ethnicity within the course.

Once this instrument is finalized, it will be proposed as a quality assurance instrument within the overall education quality assurance.

\section{CONCLUSIONS}

In order to be able to build courses that suite and motivate global learners, it is essential to develop a tool that gives instructional designers and SMEs a way to introduce gender and ethnicity during the development of mobile courses. This will enhance the learning outcomes of mobile courses because it motivates and tailors to the context and identity of different learners.

\section{ACKNOWLEDGMENT}

This instrument was the result of a collaborative work of the complete eLearning team at ITM. We are very grateful to Verena Renggli, Carlos Kiyan, Lut Lynen and Govert Van Heusden for supporting us in this endeavor.

\section{REFERENCES}

[1] M. (Ed.) Sharples Big Issues in Mobile Learning. Report of a workshop by the Kaleidoscope, Network of Excellence Mobile Learning Initiative. University of Nottingham: Kaleidoscope Research Project, 2006.

[2] A. Bandura, Self-efficacy: the exercise of control, New York: W.H. Freeman, 1997.

[3] A.L. Baylor, Y. Kim, Pedagogical Agent Design: the Impact of Agent Realism, Gender, Ethnicity, and Instructional Role, J.C. Lester et al. (Eds): ITS, LNCS 3220, Springer 2004, pp. 592-603.

[4] L. Henderson, Instructional Design of Interactive Multimedia: a Cultural Critique, ETR\&D, Vol. 44, No. 4, 1996, pp. 85 - 104, ISSN 1042-1629.

[5] I. Heemskerk, A. Brink, M. Volman, G. ten Dam, Inclusiveness and ICT in education: a focus on gender, ethnicity and social class, Journal of Computer Assisted Learning 21, 2005, pp. 1 - 16, Blackwell Publishing Ltd. (doi:10.1111/j.13652729.2005.00106.x)

\section{AUTHORS}

I. de Waard is coordinator and researcher of eLearning at the Institute of Tropical Medicine, Antwerp, Belgium (e-mail: idewaard@itg.be).

M. Zolfo, is MD and coordinator of the Telemedicine Project at the Institute of Tropical Medicine, Antwerp, Belgium (e-mail: mzolfo@itg.be).

This work was supported in part by the Belgian Directory General of Development Cooperation. Manuscript received 30 September 2008. Published as submitted by the authors. 